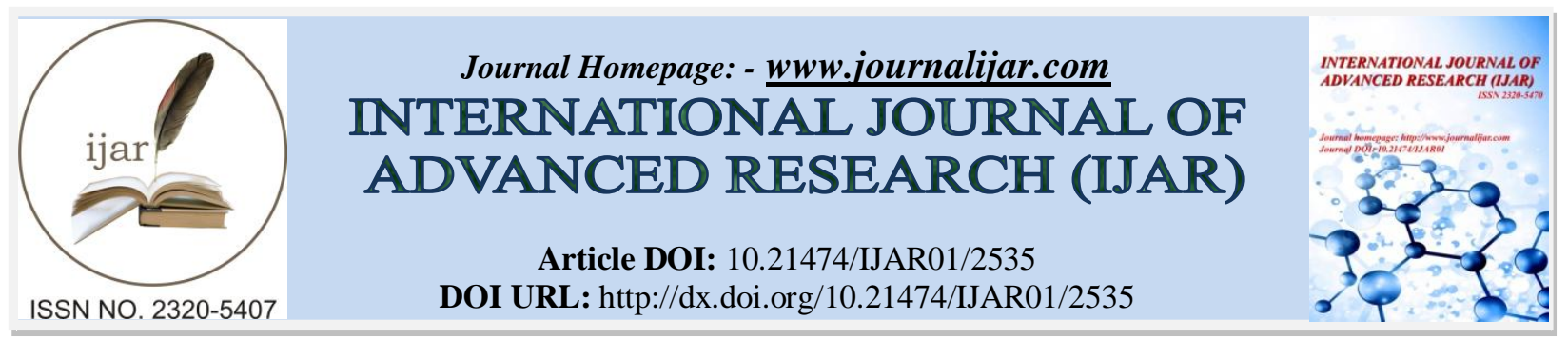

RESEARCH ARTICLE

\title{
SWEET SYNDROME IN A PATIENT WITH SYSTEMIC LUPUS ERYTHEMATOSUS FLARE.
}

\author{
"Muhammad Zeeshan Siddiqui ${ }^{1}$., Rebecca Brown ${ }^{2}$., Arezoo Haghshenas ${ }^{1}$. Qi Tao ${ }^{4}$., Samia Qazi ${ }^{1}$., Marianne \\ Frieri ${ }^{6}$. and Prachi Anand ${ }^{1}$. \\ 1. Department of internal medicine, nassau university medical center. \\ 2. New york institute of technology college of osteopathic medicine. \\ 3. Department of histopathology, nassau univeristy medical center. \\ 4. Division of allergy and immunology, department of internal medicine, nassau univeristy medical center.
}

\section{Manuscript Info}

Manuscript History

Received: 25 October 2016

Final Accepted: 23 November 2016

Published: December 2016

Key words:-

Sweet syndrome, systemic lupus erythematosus flare, erythematous plaques.

\section{Abstract}

Sweet Syndrome (SS) is an uncommon inflammatory disorder. It is accompanied by fever, leukocytosis, and abrupt appearance of cutaneous eruptions, which can be nodules, papules or plaques. We report a case of a 35-year-old Hispanic female who presented with atypical chest pain, erythematous skin lesions, elevated ESR, Ds-DNA and fever. The patient had a systemic lupus erythematosus flare with accompanying sweet syndrome, which is a very rare occurrence. Our article gives awareness to the clinicians of this rare concurrence and its knowledge will help with prompt treatment and management.

Copy Right, IJAR, 2016,. All rights reserved.

\section{Introduction:-}

Sweet Syndrome (SS) is an uncommon inflammatory disorder characterized by the abrupt appearance of fever, leukocytosis, and cutaneous eruptions, which are usually tender and erythematous papules, plaques, or nodules. Additionally, there can be involvement of the eyes, musculoskeletal system, and internal organs. Sweet syndrome is most often idiopathic but has been observed in association with a broad range of disorders, especially malignant conditions, infectious diseases, or inflammatory diseases. There are limited studies relating the development of SS with systemic lupus erythematosus (SLE).

\section{Case presentation:-}

We report a case of a 35-year-old Hispanic female who presented to the emergency department with complaints of atypical chest pain. The pain was right sided, sharp, continuous and radiating to the right shoulder. Deep inspiration aggravated the pain. As per the patient she had a fever of $101.4^{\circ} \mathrm{F}$ for last two days and dry, non-productive cough. The patient denied any recent travel or any sick contacts.

On physical examination, multiple well-demarcated, erythematous lesions were noticed on the upper extremities (Figure 1 \&2). These lesions were also appreciated on the right thigh posteromedially. Chest examination was positive for tenderness to palpation at $5^{\text {th }}$ intercostal space, along the right sternal border. The rest of the examination was unremarkable. 


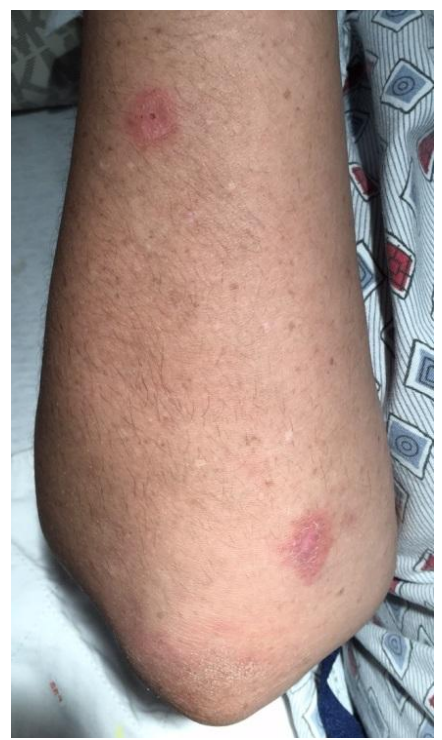

Figure 1:- Erythematous, tender maculopapular rash.

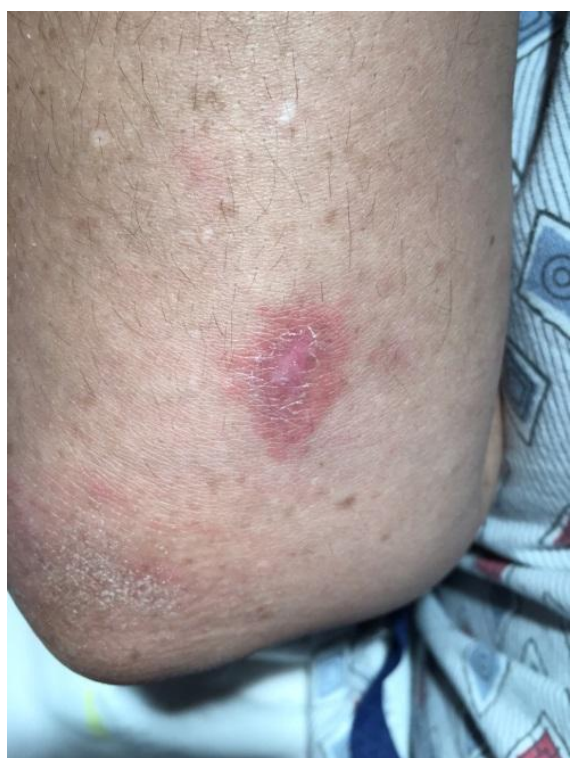

Figure 2:- Magnified view of a plaque.

The patient's past medical history is significant for SLE, Lupus nephritis (class IV-S), PE/DVT with IVC filter placement, hypertension, hyperlipidemia, and avascular necrosis (AVN) of femoral heads bilaterally which were corrected with hip arthroplasty.

An EKG was normal and laboratory examination revealed negative cardiac markers, elevated levels of ESR (61) and CRP (2.8), WBC of 9.68, platelets level of 519, Creatinine of 0.5. The c3 level was found to be 125 , C4 was $<7.5$ and Anti-dsDNA was positive with a titer of 160 . The patient was ruled out for pulmonary embolism.

On review of her medical records, the patient was found to have had a similar episode a year ago. A skin biopsy showed perivascular and interstitial dermatitis with neutrophils (Figure 3) and papillary dermal edema (Figure 4), confirming the diagnosis of Sweet Syndrome.

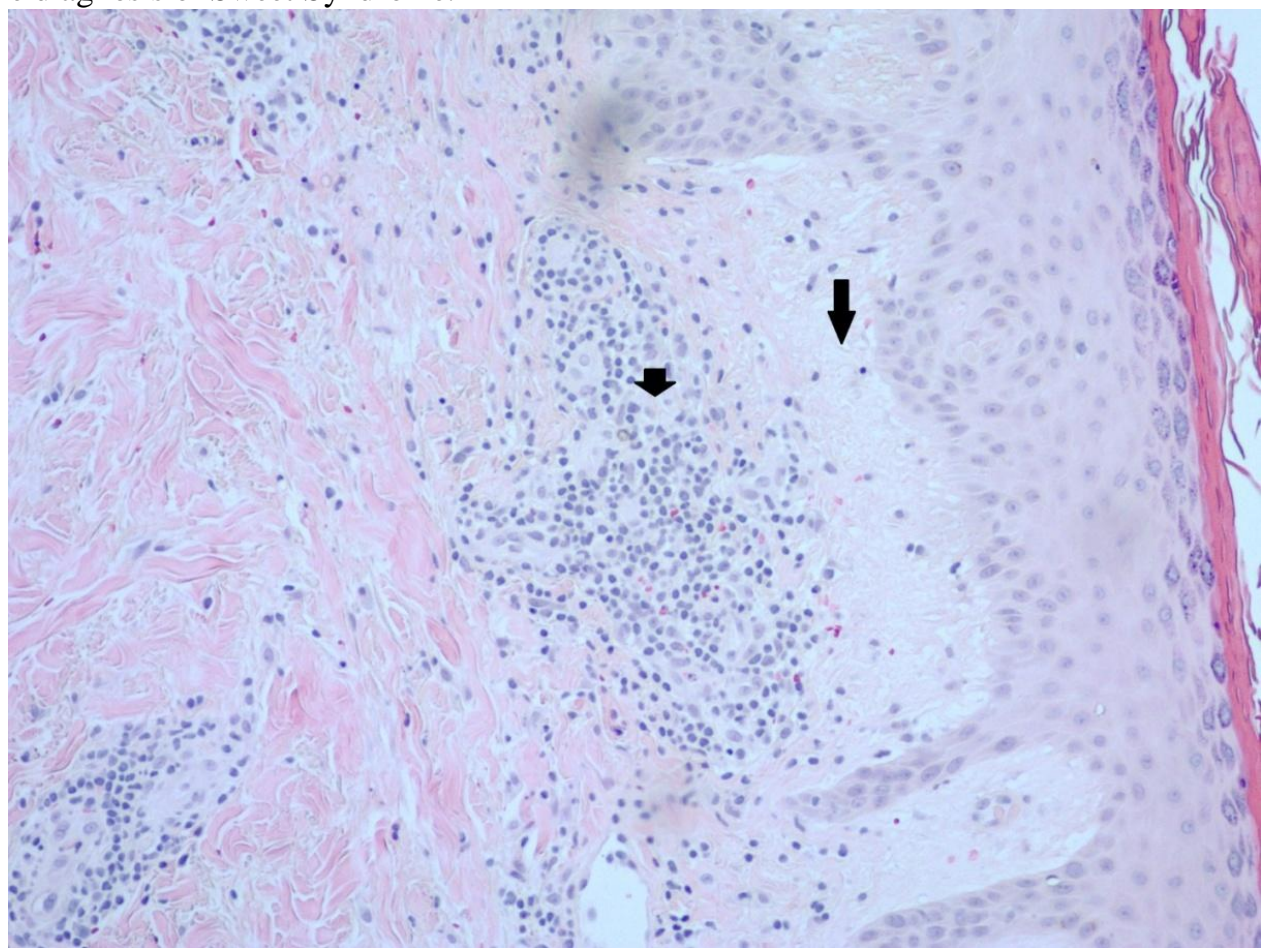

Figure 3:- Perivascular and interstitial dermatitis with neutrophils (small arrow) and papillary dermal edema (large arrow). 


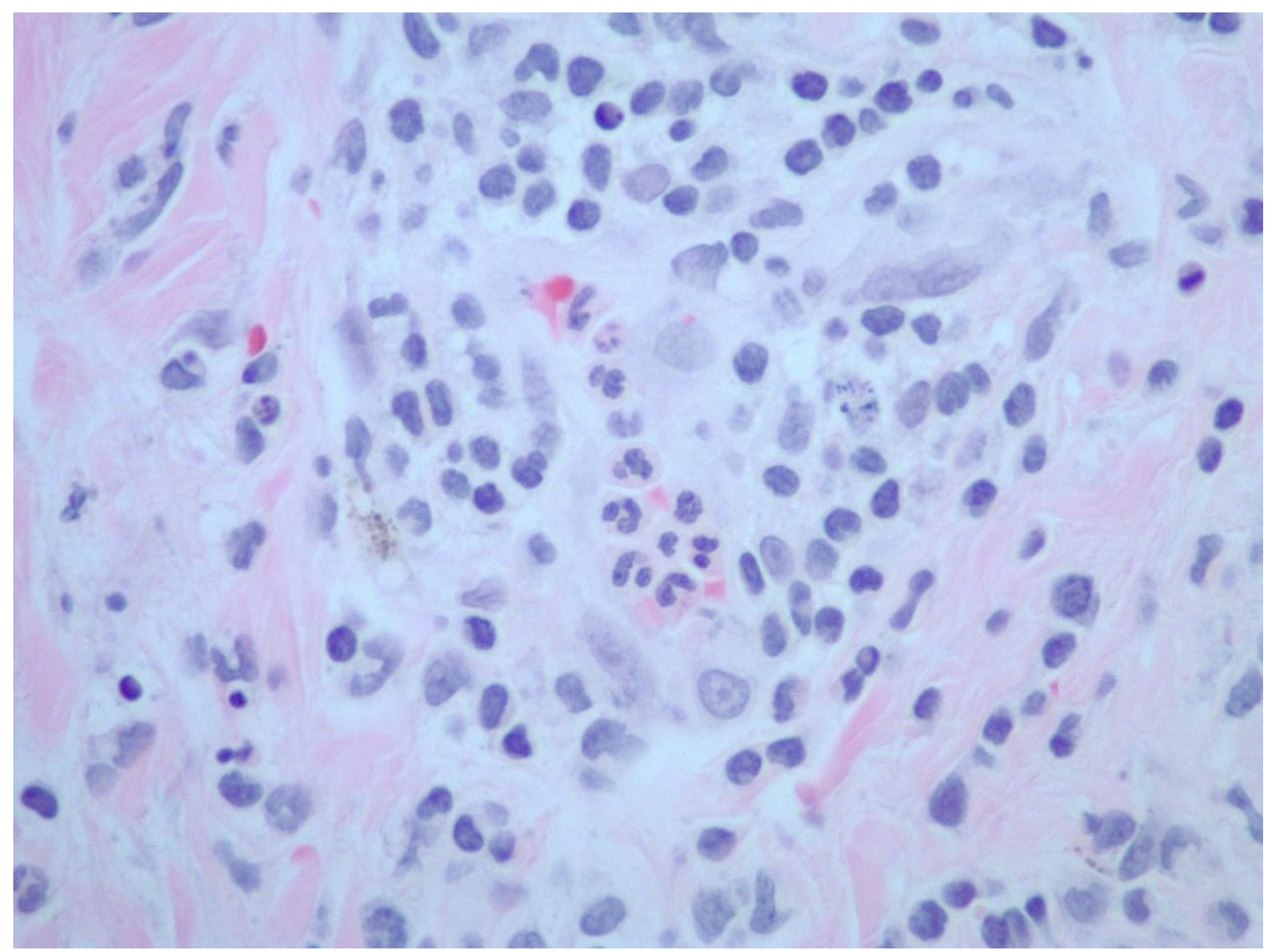

Figure 4:- Magnified view of dermatitis with neutrophilic infiltration.

The patient had been on low dose Prednisone, Hydroxychloroquine, Cellcept, and Lovenox for past 5 years. The patient was started on IV methylprednisone for Lupus flare, and over the course of time, her symptoms improved. Few cases of SS with SLE have been reported.

\section{Discussion:-}

"Sweet syndrome" is a collection of symptoms first described in 1964 by Robert Douglas Sweet after observing eight cases of women who presented with acute fever, leukocytosis, and erythematous plaques with neutrophilic infiltrates [1]. This presentation was later given its eponymous title by Whittle et al [2]. SS can present at any age, but most patients at the time of diagnosis are between 30 to 60 years of age, with 80 percent being women [3].

Cutaneous manifestations make up 3 of the 11 American College of Rheumatology (ACR) criteria to diagnose SLE, which are the classic malar rash, discoid rash or photosensitivity rash. Approximately half of the patients with SLE developed the malar rash, up to $25 \%$ develop the discoid rash and several studies have reported photosensitivity rash in 57\% to $73 \%$ of SLE patients [4]. The presence of the SS rash during an SLE flare is so rare that epidemiologic data is scarcely available to report its concurrence, however, a few studies have suggested only three cases being reported so far [5].

Etiologically, Sweet syndrome can be categorized as classical, malignancy-associated, or drug-induced. Classical Sweet syndrome includes cases that are not associated with malignancy (mostly acute myelogenous leukemia) or drug exposure (commonly granulocyte-colony stimulating factor) [3]. SS is more likely to occur in association with hematologic malignancies than solid tumor cancers [6] and usually develops about two weeks after drug exposure [7]. Most frequently associated conditions with classical SS are either infectious, inflammatory bowel disease or pregnancy. Concurrent SS and SLE are very rare. In addition, the presence of anti-La antibodies in the absence of anti-Ro antibodies proved to be another unusual part of this case. In a study by Izmirly et al., only 1.5\% of subjects with lupus were found to test positive for anti-La antibodies and test negative for anti-Ro antibodies [8].

The pathogenesis that leads to the development of Sweet syndrome is not well understood. Several factors have been theorized to contribute to the development of this disorder which includes hypersensitivity reactions, cytokine dysregulation, and genetic susceptibility. The hypersensitivity reactions may be immune mediated through 
stimulating the production of cytokines that promote neutrophil activation and infiltration [9], triggered by a variety of antigens including bacteria, viruses, medications, and malignancies. Several cytokines have been implicated in the pathogenesis of SS, of them, certain cytokines like IL-6 may also play a role in SLE through a variety of mechanisms including B cell stimulation and induction of acute phase reactants [10]. Genetically, HLA-B54 has been linked to Sweet syndrome in Japanese patients [11].

The skin lesions in SS typically have an abrupt onset, are tender, erythematous, and typically occur as papules or pustules over the head, neck, upper and lower extremities [12], though they can occur anywhere. Lesions are usually erythematous and vary in size from a few millimeters to several centimeters, and have an asymmetrical distribution. The upper extremities appear to be the most common site of involvement [12]. Skin biopsy findings of dense neutrophilic infiltrate in the dermis and sparing of the epidermis, along with prominent edema in the superficial dermis and absence of vasculitis are consistent with SS and are major diagnostic criteria of the disease. In addition to the papules, there may be other extracutaneous clinical features, musculoskeletal involvement manifesting as arthralgias, arthritis, and myalgias may be seen.

The diagnosis of Sweet syndrome is based on two major and two of four minor criteria's (Table 1) [13, 14, 15]. Our patient met almost all of the major and minor criteria required for the diagnosis of Sweet syndrome.

\begin{tabular}{|l|l|}
\hline (Table 1) Diagnostic criteria for Sweet syndrome * \\
\hline Major Criteria & $\begin{array}{l}\text { Abrupt onset of painful erythematous plaques or nodules } \\
\text { Histopathologic evidence of a dense neutrophilic infiltrates without evidence of leukocytoclastic } \\
\text { vasculitis }\end{array}$ \\
\hline $\begin{array}{l}\text { Temperature }>380 \mathrm{C} \\
\text { Association with an underlying hematologic or visceral malignancy, inflammatory disease, or } \\
\text { pregnancy, or preceded by an upper respiratory or gastrointestinal infection or vaccination } \\
\text { Excellent response to treatment with systemic corticosteroids or potassium iodide } \\
\text { Abnormal laboratory values at presentation (three of four): erythrocyte sedimentation rate }>20 \\
\mathrm{~mm} / \mathrm{hr} \text {; positive C-reactive protein; }>8,000 \text { leukocytes; }>70 \text { percent neutrophils }\end{array}$ \\
\hline
\end{tabular}

* The presence of both major criteria (1 and 2), and two of the four minor classical Sweet Syndrome.

SS has been treated with a variety of therapeutic interventions; which are primarily based on case series and individual reports of clinical experience. Corticosteroid therapy is considered first-line treatment for SS [16]. In addition, there are some reports suggesting the use of colchicine and potassium Iodide as first-line treatment options [17]. Symptoms often begin to improve within 48 hours and skin lesions usually resolve within one to two weeks after use of steroids [18]. Second-line therapies include nonsteroidal anti-inflammatory drugs (e.g., indomethacin, naproxen), dapsone, clofazimine, cyclosporine, and thalidomide. There are reports of efficacy with methotrexate, danazol, interferon-alpha, intravenous immunoglobulins, and etetrinate [17]. Lucia et al. reported successfully using rituximab for recalcitrant SS [19], but further studies are required to definitely suggest it as a treatment.

\section{Conclusion:-}

Rarely, Sweet Syndrome can be a manifestation of SLE flare. Clinicians should be aware of this rare concurrence and its knowledge will help with proper treatment and management.

\section{References:-}

1. SWEET RD. An acute febrile neutrophilic dermatosis. Br J Dermatol. 1964; 76:349.

2. Whittle CH, Beck GA, And Champion RH. Recurrent neutrophilic dermatosis of the face: a variant of Sweet's syndrome. Br J Dermatol 1968; 80:806-810.

3. Cohen PR. Sweet's syndrome--a comprehensive review of an acute febrile neutrophilic dermatosis. Orphanet $J$ Rare Dis. 2007; 2:34.

4. Millard TP, Hawk JL, McGregor JM. Photosensitivity in lupus. Lupus. 2000 Jan 1;9(1):3-10.

5. Hounoki H, Okumura M, Shinoda K, Ogawa R, Taki H, Tobe K, Shimizu K, Makino T, Shimizu T. A case of simultaneously developed sweet's syndrome and systemic lupus erythematosus in a man. International Journal of Case Reports and Images (IJCRI). 2013 May 11;3(9):36-8.

6. Raza S, Kirkland RS, Patel AA, Shortridge JR, Freter C. Insight into Sweet's syndrome and associatedmalignancy: a review of the current literature. Int J Oncol. 2013 May;42(5):1516-22. Epub 2013 Mar 28.

7. Kim MJ, Choe YH. EPONYM. Sweet syndrome. Eur J Pediatr. 2010 Dec; 169(12):1439-44. Epub 2010 May 6. 
8. Izmirly PM, Kim MY, Llanos C, Le PU, Guerra MM, Askanase AD, Salmon JE, Buyon JP. Evaluation of the risk of anti-SSA/Ro-SSB/La antibody-associated cardiac manifestations of neonatal lupus in fetuses of mothers with systemic lupus erythematosus exposed to hydroxychloroquine. Annals of the rheumatic diseases. 2010;69(10):1827

9. Voelter-Mahlknecht S, Bauer J, Metzler G, Fierlbeck G, Rassner G. Bullous variant of Sweet's syndrome. Int J Dermatol. 2005 Nov; 44(11):946-7.

10. P Youinou, C Jamin. The weight of interleukin-6 in B cell related autoimmune disorders. Journal of Autoimmunity, 2009; 32(3-4):206-210.

11. Takahama H, Kanbe T. Neutrophilic dermatosis of the dorsal hands: a case showing HLA B54, the marker of Sweet's syndrome. Int J Dermatol. 2010 Sep;49(9):1079-80.

12. Rochet NM, Chavan RN, Cappel MA, Wada DA, Gibson LE. Sweet syndrome: clinical presentation, associations, and response to treatment in 77 patients. J Am Acad Dermatol. 2013; 69(4):557.

13. von den Driesch P. Sweet's syndrome (acute febrile neutrophilic dermatosis). J Am Acad Dermatol. 1994; 31(4):535.

14. Lobo AM, Stacy R, Cestari D, Stone JH, Jakobiec FA, Sobrin L. Optic nerve involvement with panuveitis in Sweet syndrome. Ocul Immunol Inflamm. 2011;19(3):167-70.

15. Bilgin A B, Tavas P, Turkoglu E B, Ilhan H D, Toru S, Apaydin K C. An uncommon ocular manifestation of Sweet syndrome:Peripheral ulcerative keratitis and nodular scleritis. Arq Bras Oftalmol. 2015;78(1):53-5

16. Uihlein LC, Brandling-Bennett HA, Lio PA, Liang MG. Sweet syndrome in children. Pediatr Dermatol. 2012 Jan; 29(1):38-44. Epub 2011 Oct 20.

17. Schadt CR, Callen JP. Management of neutrophilic dermatoses. Dermatol Ther. 2012;25:158-172.

18. Cohen PR, Kurzrock R. Sweet's syndrome: a review of current treatment options. Am J Clin Dermatol. 2002; $3(2): 117-31$.

19. Lucia Seminario-Vidal, Guerrero C, Sami N. Refractory Sweet's syndrome successfully treated with rituximab. JAAD Case Reports 2015;1:123-5.

\section{Conflict of Interest:}

"The author(s) declare(s) that there is no conflict of interest regarding the publication of this paper." 